\title{
On general semi dynamical systems
}

\section{Peter Eris Kloeden}

In this thesis we propound a theory of multivalued dynamical systems in which no backwards extendability properties are assumed. These general semi dynamical systems are defined axiomatically in terms of attainability set functions on complete locally compact metric state spaces. This study is motivated by our use of attainability sets to model the dynamics of certain stochastic control systems and of dynamical games.

In Chapter $I$ we define and investigate as subsets of a space of random variables (mean-square convergence) the attainability sets of three stochastic control systems governed by Itô stochastic differential equations. Then elevating our results to a space of probability measures (weak convergence) we find that these attainability sets satisfy all but the backwards extendability axiom of Roxin's deterministic general control systems [2].

In Chapter II we discuss the difficulties associated with game dynamics and show that in general a player can predict the future evolution of a partie for a given initial condition only to within the attainability sets obtained by pitting his choice of strategy against the totality of admissible strategies of all the other players. Using this we suggest an attainability set based formulation of game dynamics for two-person games. In this each player is assigned a priori a set of admissible general dynamical systems interpreted in the above sense. To then reclaim the actual dynamics of the game resulting from their respective choices of general dynamical systems (that is, strategies) we take the intersection of these two general dynamical systems. We do this in terms of their common motions rather than attainable points and show that this motion-

Received 9 April 1975. Thesis submitted to the University of Queensland, September 1974. Degree approved, March 1975. Supervisors: Professor R. Výborný, Mr D.D. McGregor. 
intersection satisfies all but the backwards extendability axiom of a general dynamical system.

Fundamental properties of general semi dynamical systems are investigated in Chapter III. In particular, we establish the existence and continuity of their motions and derive a generalisation of Barbashin's theorem on the sequential compactness of their sets of motions through a given initial condition. For later use in our game model we also topologise the class of all general semi dynamical systems on a given state space and systematically develop the concept of motion-intersection of general semi dynamical systems. The topology used here is actually induced by the conjunction of four separate topological convergences, more or less to preserve the upper semicontinuity in initial conditions, continuity in time and semigroup property of general semi dynamical systems in the limit function. We show that it is at least Hausdorff.

General semi dynamical systems on compact metric spaces of probability measures are considered in Chapter IV and several examples on finite underlying spaces are given. Also we show that their motions are meaningful as stochastic processes, although they need not be Markov processes.

In Chapter $\mathrm{V}$ we axiomatically construct in terms of general semi dynamical systems a model for the dynamics of $N$-person dynamical games along the lines proposed in the second chapter. Considerable generality and technical convenience is achieved here by our using strategies only as indices of the players' admissible general semi dynamical systems. In addition we make no assumptions on the information patterns of the players. We show that this formulation of game dynamics is particularly well suited to those games in which the players have qualitative objectives and for which Lyapunov techniques can be used. In addition we give an example of a qualitative analogue of the saddle-point concept. To indicate the conceptual clarity obtainable from our model we conclude with a detailed analysis of a two-person nonzero sum quantitative game which has previously been investigated by Krasovski $Y$ and his associates on the contingent equation level [1].

Chapter VI is a systematic investigation of asymptotic invariances and eventual stabilities of sets of general semi dynamical systems. We do this partly to see their relationship with results already known in the 
literature and partly because of their usefulness for control systems and qualitative dynamical games. Necessary and sufficient Lyapunov conditions are also derived for various eventual stabilities. Our main result however is to show by counterexample that the limit sets of nonautonomous general semi dynamical systems need not be positively weakly asymptotically invariant. This greatly reduces their usefulness in classifying the behaviour of such systems.

Relative stabilities of two general semi dynamical systems are considered in Chapter VII and are used as qualitative objectives for pursuit-evasion games described by Varaiya's model [3]. We show that they are, in fact, equivalent to the corresponding Lyapunov stability of the diagonal subset of the cartesian product of state spaces with respect to the cartesian product of the two general semi dynamical systems, which we show is also a general semi dynamical system.

\section{References}

[1] H.H. Нрасовский, А.И. Субботин [N.N. KrasovskiY, A.l. Subbotin], "Дифференциальная нгра наведення" [A guidance differential game], Differencial'nye Uraunenija 6 (1970), 579-591.

[2] Emil io Roxin; "Stability in general control systems", J. Differential Equatione 1 (1965), 115-150.

[3] P.P. Varaiya, "On the existence of solutions to a differential game", SIAM J. Control 5 (1967), 153-162. 